\title{
molecules
}

ISSN 1420-3049

http://www.mdpi.org

\section{Analysis of Rotational Transitions of Methyl Formate in the Ground and First Excited Torsional States}

\author{
Hitoshi Odashima*, Kazumi Ogata, Kojiro Takagi and Shozo Tsunekawa \\ Department of Physics, Toyama University, 3190 Gofuku, Toyama 930-8555, Japan \\ * Author to whom correspondence should be addressed; e-mail: hoda@ sci.toyama-u.ac.jp
}

\begin{abstract}
The microwave spectrum of methyl formate has been observed in the 7-200 $\mathrm{GHz}$ region, and new 437 lines have been assigned to the first excited $A$ torsional substate. Both excited state lines and ground state lines reported previously were analyzed simultaneously on the basis of an internal axis method Hamiltonian. A total of 3514 lines were fitted to a 10th-order reduced Hamiltonian model involving 67 molecular parameters to a $1 \sigma$ standard deviation of $179 \mathrm{kHz}$.
\end{abstract}

Keywords: Methyl formate, $\mathrm{HCOOCH}_{3}$, microwave spectrum, rotational transition, internal axis method, interstellar molecule.

\section{Introduction}

Methyl formate $\left(\mathrm{HCOOCH}_{3}\right)$ is an interesting molecule in molecular spectroscopy, because a large amplitude torsional vibration makes it difficult to reproduce observed torsion-rotation transition frequencies as accurately as those of a typical semirigid molecule. This molecule is also of astronomical interest, because 391 rotational transitions in its ground torsional state have been observed in star forming regions such as Sgr B2 and OMC-1[1]. The microwave spectrum of this molecule has been observed up to $608 \mathrm{GHz}$, and rotational transitions in the ground torsional state have been extensively analyzed by several researchers [2-9]. For excited torsional states, however, no transition has been assigned as far as we know. An analysis of rotational transitions in excited torsional states is important to determine an effective Hamiltonian of this molecule, since many molecular parameters of the Hamiltonian can only become free of correlation when data from excited torsional states is available. An 
analysis of excited state lines is also important to identify astrophysical unidentified lines. Star forming regions where the existence of methyl formate has been confirmed are relatively hot and many unidentified lines from these regions are supposed to be excited torsional state lines of methyl formate.

In this study, we have observed the microwave spectrum of methyl formate in the 7- $200 \mathrm{GHz}$ region and assigned new 437 lines to the first excited $A$ torsional substate. Both the first excited torsional state lines and the ground state lines reported previously [2-9] were analyzed simultaneously on the basis of an internal axis method (IAM) Hamiltonian [10]. A total of 3514 lines were fitted to a $1 \sigma$ standard deviation of $179 \mathrm{kHz}$, and molecular parameters were determined.

\section{Experimental Procedure and Assignments}

The microwave spectrum of methyl formate has been observed in the 7-200 GHz region. A part of the observed spectrum is shown in Figure1. A synthesized sweeper $(<40 \mathrm{GHz})$ was used as a microwave source, which was combined with microwave amplifiers and multipliers to provide complete coverage of the 7-200 GHz region. More than 40000 lines have been observed in this region with a typical accuracy of $50 \mathrm{kHz}$. Details on our microwave spectrometer have been described elsewhere [11].

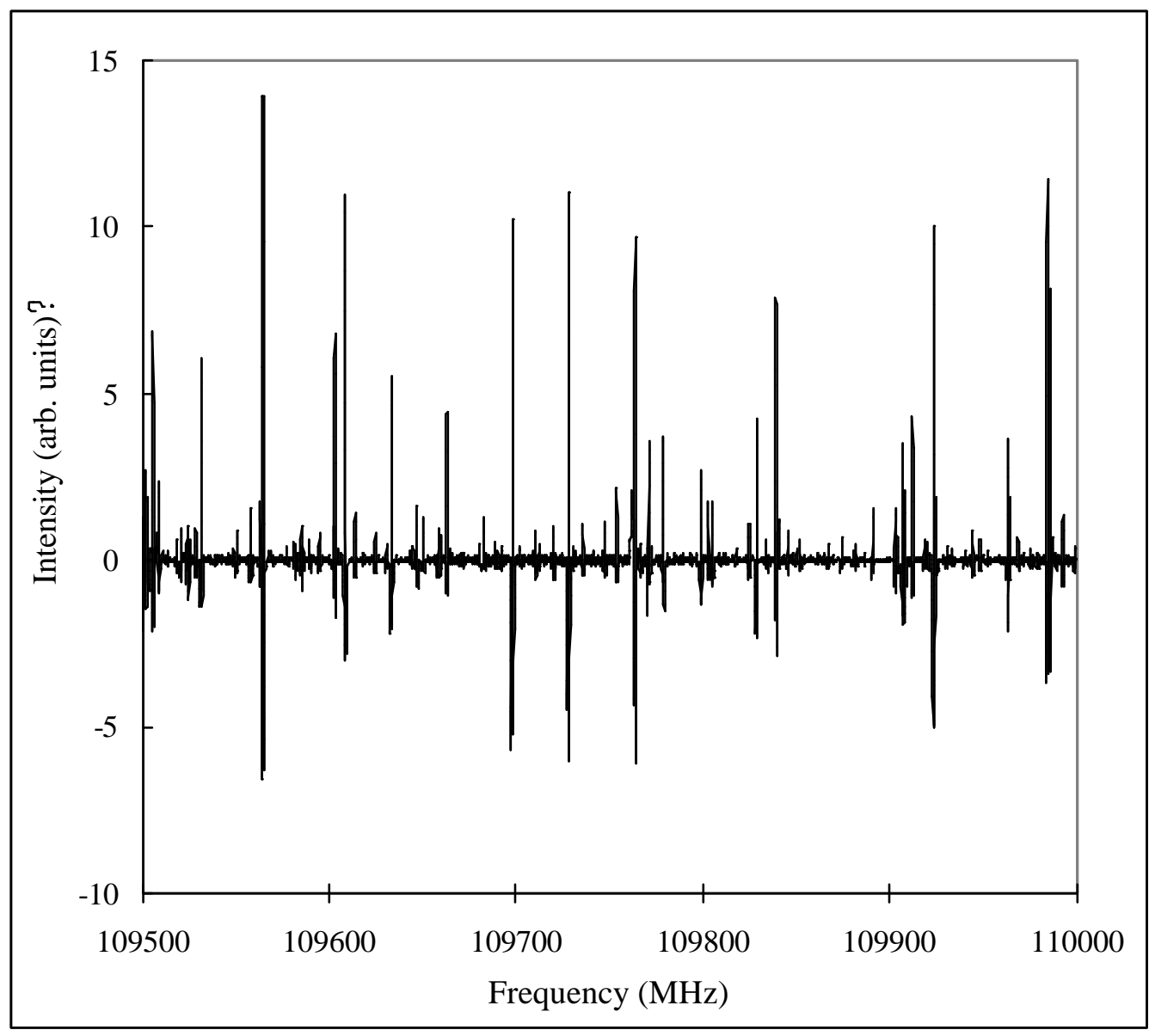

Figure 1. A Part of the Observed Spectrum. 
We searched first excited torsional state lines (satellite lines) around the corresponding ground state lines (main lines) assigned previously, taking intensities of satellite lines into account. The intensity of a satellite line is about half of that of a main line due to the torsional energy difference of $\sim 130 \mathrm{~cm}^{-1}$ [12]. Satellite lines from low- $J$ transitions were assigned by observing Stark effects. For $A$-species, satellite lines including higher- $J$ transitions were fitted to a Watson $A$-reduced Hamiltonian by neglecting the effect of methyl torsion. We assigned $437 A$-species lines with $J \leq 17$ and $K_{a} \leq 7$ to the first excited torsional state, and confirmed our assignments by forming closed transition frequency loops; each loop consists of four observed frequencies. No $E$-species line was, however, assigned definitely because the number of assigned transitions was insufficient for combination relations. The distrib ution of errors of loops for the excited torsional state is shown in Figure 2. Although several loops have errors of near 1 $\mathrm{MHz}$ because of uncertainties due to blended lines, the $1 \sigma$ standard deviation of the errors of loops is about $100 \mathrm{kHz}$. This is consis tent with our estimate of the experimental uncertainty of $50 \mathrm{kHz}$ for a line, because the law of propagation of errors gives an uncertainty of $100 \mathrm{kHz}$ to each loop which consists of four frequencies.

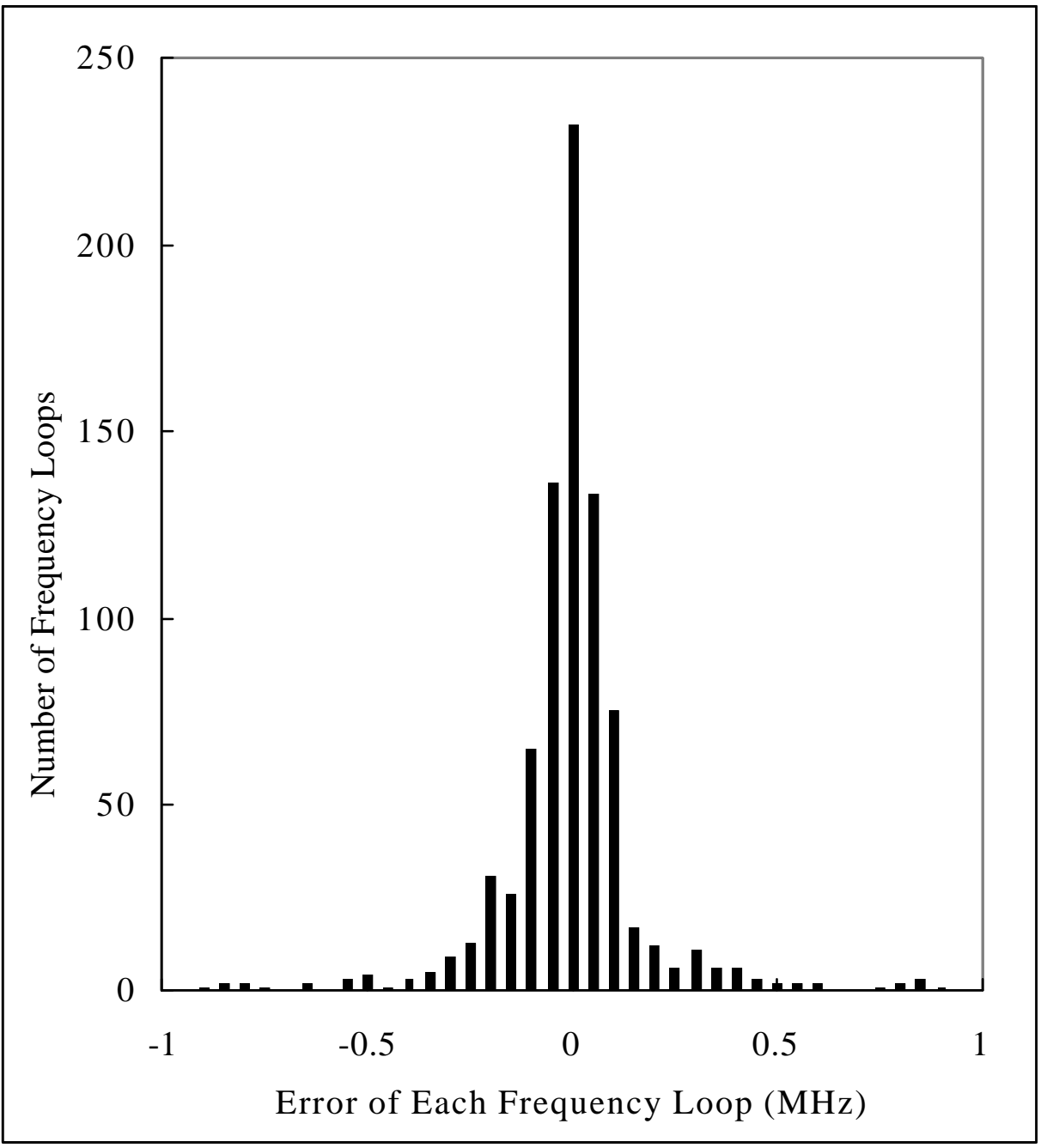

Figure 2. Histogram of Errors of Frequency Loops. 


\section{Analysis and Discussion}

In the previous analysis of the ground state lines [9], we successfully used a 10th-order reduced IAM Hamiltonian with 53 parameters, which includes the term $D_{a b 2}\left(P_{a} P_{b}+P_{b} P_{a}\right) P^{2}$, a correction term to the $D_{a b}$ term. For methyl formate, the $\mathrm{CH}_{3}$-symmetry axis differs by around $52^{\circ}$ from the principal $a$-axis and the value of $\left|D_{a b}\right|$ is large [8]. Furthermore, methyl formate has low energy vibrational modes such as COC deformation $\left(318 \mathrm{~cm}^{-1}\right)$ and $\mathrm{C}-\mathrm{O}$ torsion $\left(332 \mathrm{~cm}^{-1}\right)$ which possibly contribute much to the centrifugal distortion effect [12]. In reduction of a Hamiltonian, the $D_{a b 2}$ term should be retained to make a large correction to the $D_{a b}$ term for the centrifugal distortion. In the present study, we simultaneous ly analyzed rotational transitions of the first excited and ground torsional states on the basis of the same Hamiltonian model. For this simultaneous analysis, we needed 14 more parameters which were set to zero in the previous analysis [9]. The parameters used are defined in Table 1.

Our data set consists of a total of 3514 lines, including both 437 newly assigned $A$-species lines with $J \leq 17$ and $K_{a} \leq 7$ of the first excited torsional state and 3077 previously reported $A$ - and $E$-species lines with $J \leq 43$ and $K_{a} \leq 18$ of the ground state. We limited our data set to transitions whose assignments were confirmed by combination relations, excluding $\sim 500$ lines with higher $J$ and $K_{a}$ reported in Ref. [8]. The same weight was given to all the observed frequencies, because their experimental uncertainties are comparable in magnitude $(\sim 100 \mathrm{kHz})$. Details on the fitting procedure have been reported elsewhere [13].

The data set was fitted to the Hamiltonian model involving 67 parameters to a $1 \sigma$ standard deviation of $179 \mathrm{kHz}$. Determined molecular parameters are shown in Table 2. The parameter $F$ is fixed to the value obtained previously because of its strong correlation with the parameter $\rho$ [7]. The absolute value of the parameter $D_{a b 2}$ is comparable with those of other quadratic centrifugal distortion parameters, and makes a noticeable contribution to the calculated transition frequencies even for medium- $J$ transitions. We added 14 parameters, $k_{7}, V_{6}, c_{9}, c_{19}, N_{V}, K_{2}, c_{11}, d d_{a b}, h_{J 6}, n_{V}, v_{V}, n_{K}, c_{13}$, and $c_{16}$, to the Hamiltonian to fit the data set with the same quality as was obtained in the previous analysis of the ground state lines. It is noted that most of these parameters are the coefficients for coupling terms between rotational angular momenta and torsional potential. Data from the first excited torsional state has become available, and the information on torsional dependence of rotational energy levels has been obtained in the present study. Therefore, the parameters for coupling terms were determined. Since we included the $D_{a b 2}$ term in the Hamiltonian, one of the $k_{7}, k_{6}$ ', and $V_{6}$ terms should be removed to keep the Hamiltonian completely reduced [14]. In this analysis, we removed the $k_{6}$ ' term and therefore the Hamiltonian remains a completely reduced Hamiltonian.

In summary, the 10th-order reduced IAM Hamiltonian model including the $D_{a b 2}$ term has been successfully used to analyze rotational transitions of the ground state and the first excited $A$ torsional substate of methyl formate. The next step in this work is an analysis of $E$-species lines of the first excited torsional state. Assignments of these lines are in progress, according to frequencies calculated from the molecular parameters determined in this work. 
Table 1. IAM Reduced Hamiltonian Parameters for Methyl Formate

\begin{tabular}{|c|c|c|c|c|c|c|}
\hline \multirow{2}{*}{ Rotational Operator } & \multicolumn{6}{|c|}{ Torsional Operator } \\
\hline & 1 & $\mathrm{P}_{v}^{2}$ & $\mathrm{P}_{\gamma} \mathrm{P}_{\mathrm{a}}$ & $\cos 3 \gamma$ & $\left\{\mathrm{P}_{\gamma}^{2}, \cos 3 \gamma\right\}$ & $\cos 6 y$ \\
\hline 1 & & $\mathrm{~F}$ & & $-V_{3} / 2$ & $\mathrm{k}_{7}$ & $-\mathrm{V}_{6} / 2$ \\
\hline $\mathrm{P}^{2}$ & $(\mathrm{~B}+\mathrm{C}) / 2$ & & $\mathrm{~L}_{\mathbf{V}}$ & $F_{V}$ & & $\mathrm{~N}_{\mathrm{v}}$ \\
\hline $\mathrm{P}_{\mathrm{a}}^{2}$ & $A-(B+C) / 2$ & & & $\mathrm{~K}_{5}$ & & $\mathrm{~K}_{2}$ \\
\hline $\mathrm{P}_{\mathrm{b}}^{2}-\mathrm{P}_{\mathrm{c}}^{2}$ & $(\mathrm{~B}-\mathrm{C}) / 2$ & & & $c_{2}$ & $c_{19}$ & $c_{11}$ \\
\hline$\left\{P_{a}, P_{b}\right\}$ & $\mathrm{D}_{\mathrm{ab}}$ & & & $d_{a b}$ & & $\mathrm{dd}_{\mathrm{ab}}$ \\
\hline $\mathrm{P}^{4}$ & $-\Delta_{\mathrm{J}}$ & & $l_{V}$ & $\mathrm{f}_{\mathrm{V}}$ & & $\mathrm{n}_{\mathrm{V}}$ \\
\hline $\mathrm{P}^{2} \mathrm{~Pa}^{2}$ & $-\Delta_{\mathrm{JK}}$ & & & $\phi_{\mathbf{v}}$ & & $v_{\mathbf{v}}$ \\
\hline $\mathrm{P}_{\mathrm{a}}^{4}$ & $-\Delta_{K}$ & & & $\mathrm{f}_{\mathrm{K}}$ & & $\mathrm{n}_{\mathrm{K}}$ \\
\hline $2 \mathrm{P}^{2}\left(\mathrm{P}_{\mathrm{b}}^{2}-\mathrm{P}_{\mathrm{c}}^{2}\right)$ & $-\delta_{\mathrm{J}}$ & & & $\mathrm{c}_{6}$ & & $c_{13}$ \\
\hline$\left\{\mathrm{P}_{\mathrm{a}}^{2},\left(\mathrm{P}_{\mathrm{b}}^{2}-\mathrm{P}_{\mathrm{c}}^{2}\right)\right\}$ & $-\delta_{K}$ & & & $\mathrm{C}_{9}$ & & $c_{16}$ \\
\hline $\mathrm{P}^{2}\left\{\mathrm{P}_{\mathrm{a}}, \mathrm{P}_{\mathrm{h}}\right\}$ & $\mathrm{D}_{\mathrm{ah2}}$ & & & & & \\
\hline$P^{6}$ & $\mathrm{H}_{\mathrm{J}}$ & & $\mathrm{h}_{\mathrm{I} 6 \mathrm{G}}$ & $h_{I / 4}$ & & \\
\hline $\mathrm{P}^{4} \mathrm{P}_{\mathrm{a}}^{2}$ & $\mathrm{H}_{\mathrm{JK}}$ & & $h_{\mathbf{J 3}}$ & $h_{J 1}$ & & \\
\hline $\mathrm{P}^{2} \mathrm{P}_{\mathrm{a}}^{4}$ & $\mathrm{H}_{\mathrm{KJ}}$ & & & & & \\
\hline $\mathrm{P}_{\mathrm{a}}{ }^{6}$ & $\mathrm{H}_{\mathrm{K}}$ & & & & & \\
\hline $2 \mathrm{P}^{4}\left(\mathrm{P}_{\mathrm{b}}^{2}-\mathrm{P}_{\mathrm{c}}^{2}\right)$ & $\mathrm{h}_{\mathrm{I}}$ & & & $c_{27}$ & & \\
\hline $\mathrm{P}^{2}\left\{\mathrm{P}_{\mathrm{a}}^{2},\left(\mathrm{P}_{\mathrm{b}}^{2}-\mathrm{P}_{\mathrm{c}}^{2}\right)\right\}$ & $\mathrm{h}_{\mathrm{JK}}$ & & & & & \\
\hline$\left\{\mathrm{P}_{\mathrm{a}}^{4},\left(\mathrm{P}_{\mathrm{b}}{ }^{2}-\mathrm{P}_{\mathrm{c}}{ }^{2}\right)\right\}$ & $\mathrm{h}_{\mathbf{K}}$ & & & & & \\
\hline$P^{4}\left\{P_{a}, P_{b}\right\}$ & $\mathrm{DD}_{\mathrm{ab}}$ & & & & & \\
\hline $\mathrm{P}^{8}$ & $\mathrm{~L}_{\mathrm{J}}$ & & & & & \\
\hline $\mathrm{P}^{6} \mathrm{P}_{\mathrm{a}}^{2}$ & $\mathrm{~L}_{\text {.J.JK }}$ & & & & & \\
\hline $\mathrm{P}^{4} \mathrm{P}_{\mathrm{a}}^{4}$ & $\mathrm{~L}_{\mathbf{J K}}$ & & & & & \\
\hline $\mathrm{P}^{2} \mathrm{P}_{\mathrm{a}}^{6}$ & $\mathrm{~L}_{\mathrm{JKK}}$ & & & & & \\
\hline & $\mathrm{L}_{\mathrm{K}}$ & & & & & \\
\hline $2 \mathrm{P}^{6}\left(\mathrm{P}_{\mathrm{b}}^{2}-\mathrm{P}_{\mathrm{c}}^{2}\right)$ & $l_{J}$ & & & & & \\
\hline $\mathrm{P}^{4}\left\{\mathrm{P}_{\mathrm{a}}^{2},\left(\mathrm{P}_{\mathrm{b}}^{2}-\mathrm{P}_{\mathrm{c}}^{2}\right)\right\}$ & $1_{\mathrm{JK}}$ & & & & & \\
\hline $\mathrm{P}^{2}\left\{\mathrm{P}_{\mathrm{a}}^{4},\left(\mathrm{P}_{\mathrm{b}}{ }^{2}-\mathrm{P}_{\mathrm{c}}{ }^{2}\right)\right\}$ & $1_{\text {K.I }}$ & & & & & \\
\hline$\left\{\mathrm{P}_{\mathrm{a}}^{6},\left(\mathrm{P}_{\mathrm{b}}{ }^{2}-\mathrm{P}_{\mathrm{c}}{ }^{2}\right)\right\}$ & $1_{K}$ & & & & & \\
\hline $\mathrm{P}^{10}$ & $P_{. J}$ & & & & & \\
\hline $\mathrm{P}^{8} \mathrm{P}_{\mathrm{a}}^{2}$ & & & & & & \\
\hline $\mathrm{P}^{6} \mathrm{P}_{\mathrm{a}}^{4}$ & $P_{J K}$ & & & & & \\
\hline $\mathrm{P}^{4} \mathrm{P}_{\mathrm{a}}^{6}$ & $P_{K . J}$ & & & & & \\
\hline $\mathrm{P}^{2} \mathrm{P}_{\mathrm{a}}^{8}$ & $\mathrm{P}_{\mathrm{JKK}}$ & & & & & \\
\hline $\mathrm{P}_{\mathrm{a}}^{10}$ & $\mathrm{P}_{\mathrm{K}}$ & & & & & \\
\hline $2 \mathrm{P}^{8}\left(\mathrm{P}_{\mathrm{b}}^{2}-\mathrm{P}_{\mathrm{c}}^{2}\right)$ & $\mathrm{p}_{\mathrm{I}}$ & & & & & \\
\hline $\mathrm{P}^{6}\left\{\mathrm{P}_{\mathrm{a}}^{2},\left(\mathrm{P}_{\mathrm{b}}^{2}-\mathrm{P}_{\mathrm{c}}^{2}\right)\right\}$ & $\mathrm{p}_{\mathrm{JJK}}$ & & & & & \\
\hline $\mathrm{P}^{4}\left\{\mathrm{P}_{\mathrm{a}}{ }^{4},\left(\mathrm{P}_{\mathrm{b}}{ }^{2}-\mathrm{P}_{\mathrm{c}}{ }^{2}\right)\right\}$ & рЈк $_{1}$ & & & & & \\
\hline $\mathrm{P}^{2}\left\{\mathrm{P}_{\mathrm{a}}{ }^{6},\left(\mathrm{P}_{\mathrm{b}}{ }^{2}-\mathrm{P}_{\mathrm{c}}{ }^{2}\right)\right\}$ & & & & & & \\
\hline$\left\{\mathrm{P}_{\mathrm{a}}^{8},\left(\mathrm{P}_{\mathrm{b}}^{2}-\mathrm{P}_{\mathrm{c}}^{2}\right)\right\}$ & $\mathrm{p}_{\mathrm{K}}$ & & & & & \\
\hline
\end{tabular}


Table 2. Determined Molecular Parameters of Methyl Formate

\begin{tabular}{|c|c|c|c|c|c|}
\hline Parameter & Value [MHz] & & Parameter & Value [MHz] & \\
\hline $\mathrm{A}-(\mathrm{B}+\mathrm{C}) / 2$ & $1.196392(78)$ & $\times 10^{4}$ & $\mathrm{c}_{19}$ & $5.787(48)$ & $\times 10^{-1}$ \\
\hline$(\mathrm{B}+\mathrm{C}) / 2$ & $7.20460(14)$ & $\times 10^{3}$ & $\mathrm{~N}_{\mathrm{V}}$ & $-3.5618(82)$ & $\times 10^{0}$ \\
\hline$(\mathrm{B}-\mathrm{C}) / 2$ & $1.92152(22)$ & $\times 10^{3}$ & $\mathrm{~K}_{2}$ & $1.1899(69)$ & $\times 10^{2}$ \\
\hline $\mathrm{D}_{\mathrm{ab}}$ & $-5.19966(24)$ & $\times 10^{3}$ & $c_{11}$ & $-2.1095(48)$ & $\times 10^{1}$ \\
\hline F [fixed] & 1.68142 & $\times 10^{5}$ & $\mathrm{dd}_{\mathrm{ab}}$ & $-2.1872(48)$ & $\times 10^{1}$ \\
\hline$\rho$ [unitless] & $8.39818(70)$ & $\times 10^{-2}$ & $\mathrm{~L}_{\mathbf{J}}$ & $-3.4662(73)$ & $\times 10^{-10}$ \\
\hline $\mathrm{V}_{3}\left[\mathrm{~cm}^{-1}\right]$ & $3.80434(44)$ & $\times 10^{2}$ & $\mathrm{~L}_{\text {JJK }}$ & $2.3592(49)$ & $\times 10^{-9}$ \\
\hline$\Delta_{\mathrm{J}}$ & $1.19198(57)$ & $\times 10^{-1}$ & $\mathrm{~L}_{\mathbf{J K}}$ & $-2.8943(41)$ & $\times 10^{-8}$ \\
\hline$\Delta_{\mathrm{JK}}$ & $-8.3133(44)$ & $\times 10^{-1}$ & $\mathrm{~L}_{\mathbf{J K K}}$ & $4.1668(62)$ & $\times 10^{-8}$ \\
\hline$\Delta_{\mathrm{K}}$ & $8.3260(37)$ & $\times 10^{-1}$ & $\mathrm{~L}_{\mathrm{K}}$ & $-3.3118(55)$ & $\times 10^{-8}$ \\
\hline$\delta_{\mathbf{J}}$ & $5.0065(27)$ & $\times 10^{-2}$ & $l_{\mathbf{J}}$ & $-1.5480(27)$ & $\times 10^{-10}$ \\
\hline$\delta_{\mathrm{K}}$ & $-8.2476(44)$ & $\times 10^{-2}$ & $1_{\mathrm{JK}}$ & $1.2004(30)$ & $\times 10^{-10}$ \\
\hline $\mathrm{D}_{\mathrm{ab2} 2}$ & $5.6677(56)$ & $\times 10^{-2}$ & $1_{\mathrm{KJ}}$ & $-2.0012(41)$ & $\times 10^{-9}$ \\
\hline $\mathrm{L}_{\mathbf{V}}$ & $6.253(27)$ & $\times 10^{-1}$ & $1_{K}$ & $2.3439(60)$ & $\times 10^{-9}$ \\
\hline $\mathrm{F}_{\mathbf{V}}$ & $1.0730(12)$ & $\times 10^{2}$ & $h_{J 6}$ & $-4.973(13)$ & $\times 10^{-11}$ \\
\hline $\mathrm{k}_{5}$ & $-6.163(11)$ & $\times 10^{2}$ & $h_{J 3}$ & $-1.7708(35)$ & $\times 10^{-7}$ \\
\hline$c_{2}$ & $8.304(18)$ & $\times 10^{1}$ & $h_{J 4}$ & $-9.577(19)$ & $\times 10^{-6}$ \\
\hline$d_{a b}$ & $2.1490(23)$ & $\times 10^{2}$ & $\mathrm{~h}_{\mathrm{J} 1}$ & $4.6263(74)$ & $\times 10^{-5}$ \\
\hline $\mathrm{k}_{7}$ & $9.2(78)$ & $\times 10^{1}$ & $c_{27}$ & $-5.1951(98)$ & $\times 10^{-6}$ \\
\hline $\mathrm{V}_{6}\left[\mathrm{~cm}^{-1}\right]$ & $2.5166(97)$ & $\times 10^{1}$ & $\mathrm{n}_{\mathrm{v}}$ & $-5.654(12)$ & $\times 10^{-3}$ \\
\hline $\mathrm{H}_{\mathbf{J}}$ & $1.2361(17)$ & $\times 10^{-5}$ & $v_{v}$ & $-4.2392(79)$ & $\times 10^{-2}$ \\
\hline $\mathrm{H}_{\mathbf{J K}}$ & $-2.7463(52)$ & $\times 10^{-5}$ & $\mathrm{n}_{\mathrm{K}}$ & $5.133(12)$ & $\times 10^{-2}$ \\
\hline $\mathrm{H}_{\mathrm{KJ}}$ & $-1.4411(13)$ & $\times 10^{-4}$ & $\mathrm{c}_{13}$ & $3.9907(65)$ & $\times 10^{-3}$ \\
\hline $\mathrm{H}_{\mathrm{K}}$ & $1.5737(13)$ & $\times 10^{-4}$ & $c_{16}$ & $-1.6248(37)$ & $\times 10^{-2}$ \\
\hline $\mathrm{h}_{\mathrm{J}}$ & $6.5035(82)$ & $\times 10^{-6}$ & $P_{\mathbf{J}}$ & $1.545(17)$ & $\times 10^{-14}$ \\
\hline$h_{J K}$ & $6.5763(87)$ & $\times 10^{-6}$ & $P_{J K}$ & $-1.3287(31)$ & $\times 10^{-12}$ \\
\hline $\mathrm{h}_{\mathrm{K}}$ & $-1.3274(13)$ & $\times 10^{-5}$ & $\mathrm{P}_{\mathrm{KJ}}$ & $6.583(15)$ & $\times 10^{-12}$ \\
\hline $\mathrm{DD}_{\mathrm{ab}}$ & $3.5966(87)$ & $\times 10^{-7}$ & $P_{\text {JKK }}$ & $-1.3322(31)$ & $\times 10^{-11}$ \\
\hline$l_{\mathbf{V}}$ & $4.705(11)$ & $\times 10^{-5}$ & $\mathrm{P}_{\mathrm{K}}$ & $8.328(19)$ & $\times 10^{-12}$ \\
\hline$f_{v}$ & $1.9131(30)$ & $\times 10^{-2}$ & $\mathrm{p}_{\mathbf{J}}$ & $1.911(16)$ & $\times 10^{-15}$ \\
\hline$\phi_{\mathbf{v}}$ & $9.989(23)$ & $\times 10^{-3}$ & $\mathrm{p}_{\mathrm{JJK}}$ & $7.6242(38)$ & $\times 10^{-14}$ \\
\hline$f_{K}$ & $-9.321(22)$ & $\times 10^{-3}$ & $\mathrm{p}_{\mathrm{JK}}$ & $-7.9465(84)$ & $\times 10^{-14}$ \\
\hline$c_{6}$ & $-4.4934(98)$ & $\times 10^{-3}$ & $\mathrm{p}_{\mathrm{K}}$ & $2.7938(36)$ & $\times 10^{-13}$ \\
\hline$c_{9}$ & $1.0917(26)$ & $\times 10^{-2}$ & $\sigma[\mathrm{kHz}]$ & 179 & \\
\hline
\end{tabular}




\section{Acknowledgments}

The authors thank Mr. Yamada for his help in the analysis. This work was partly supported by a Grant-in-Aid from the Ministry of Education, Science, Sports and Culture of Japan. The authors thank the Computer Center of the Institute for Molecular Science, for the use of the Fujitsu VPP5000 and NEC SX-5 computers. The calculation was also carried out at Computing and Network Services of Toyama University.

\section{References}

1. Lovas, F. J. J. Phys. Chem. Ref. Data, 1992, 21, 181-272.

2. Curl, R. F. J. Chem. Phys. 1959, 30, 1529-1536.

3. Brown, R. D.; Crofts, J. G.; Gardner, F. F.; Gordfrey, P. D.; Robinson, B. J.; Whiteoak, J. B. Astrophys. J. 1975, 197, L29-L31.

4. Bauder, A. J. Phys. Chem. Ref. Data, 1979, 8, 583-618.

5. DeMaison, J.; Boucher, D.; Dubrulle, A.; Van Eijck, B. P. J. Mol. Spectrosc. 1983, 102, 260-263.

6. Plummer, G. M.; Herbst, E.; De Lucia, F. C.; Blake, G. A. Astrophys. J. Suppl. 1984, 55, 633-656.

7. Plummer, G. M.; Herbst, E.; De Lucia, F. C.; Blake, G. A. Astrophys. J. Suppl. 1986, 60, 949-961.

8. Oesterling, L. C.; Albert, S.; De Lucia, F. C.; Sastry, K. V. L. N.; Herbst, E. Astrophys. J. 1999 521, 255-260; its electronic edition.

9. Karakawa, Y.; Oka, K.; Odashima, H.; Takagi, K.; Tsunekawa, S. J. Mol. Spectrosc. 2001, 210, 196-212.

10. Lees, R. M.; Baker, J. G. J. Chem. Phys. 1968, 48, 5299-5318.

11. Fukuyama, Y.; Odashima, H.; Takagi, K.; Tsunekawa, S. Astrophys. J. Suppl. 1996, 104, 329-346.

12. Shimanouchi, T. Tables of Molecular Vibrational Frequencies Consolidated Volume I, National Bureau of Standards, 1972, 1-160

13. Odashima, H.; Matsushima, F.; Nagai, K.; Tsunekawa, S.; Takagi, K. J. Mol. Spectrosc. 1995, 173, 404-422.

14. Tang, J.; Takagi, K. J. Mol. Spectrosc. 1993, 161, 487-498.

(C) 2003 by MDPI (http://www.mdpi.org). Reproduction is permitted for noncommercial purposes. 